\title{
Surgical and Locoregional Therapy of HCC: TACE
}

\author{
Masakatsu Tsurusaki Takamichi Murakami \\ Department of Radiology, Kinki University, Faculty of Medicine, Osaka, Japan
}

\section{Key Words}

Chemoembolization · Drug eluting bead - Gelatin sponge $\cdot$ Hepatocellular carcinoma

Iodized oil

\begin{abstract}
Transcatheter arterial chemoembolization (TACE) is performed worldwide for patients with intermediate-stage hepatocellular carcinoma (HCC). TACE has produced survival advantages in two randomized controlled trials and a meta-analysis, and is currently the mainstay of treatment for this stage of HCC. However, there are currently no global guidelines regarding the dose, choice or combination of cytotoxic agents for TACE; therefore, it is difficult to compare data from different TACE studies. In Japan, most of the TACE procedures have been based on iodized oil as conventional TACE, utilizing the microembolic and drug-carrying characteristic of iodized oil. Superselective TACE with lipiodol is the primary TACE procedure that has reported satisfactory levels of local control associated with a lower risk of complications. Conversely, TACE performed using drug-eluting beads has been widely used in western countries, and this has shown similar tumor response and median survival compared to conventional TACE. Moreover, the combination of TACE and molecular targeted agents is now ongoing to evaluate the synergistic effect. In this review, the indication, technical issues, and complications of TACE are reviewed.
\end{abstract}

Copyright $@ 2015$ S. Karger AG, Basel

\section{Introduction}

Hepatocellular carcinoma (HCC) is the fifth leading cause of cancer-related deaths worldwide [1]. Currently, many cases of HCC are detected at an early stage as a result of screening of high-risk patients. Conventionally, hepatectomy and liver transplantation were 
considered to be the only therapeutic options with the potential to achieve a complete cure. However, only between $9 \%-29 \%$ of patients will benefit from hepatectomy because of the high incidence of complications due to chronic liver disease.

Transcatheter arterial chemoembolization (TACE) is widely performed globally as an effective treatment for inoperable HCC. In 1977, Yamada et al. developed transarterial embolization (TAE) [2]; thereafter, an emulsion of lipiodol (Lipiodol UltraFluid $®$, Andre Guerbet, Aulnay-sous-Bois, France) and an chemotherapeutic agent were added that led to the creation of TACE [3-11]. TACE with a lipiodol-chemotherapeutic agent suspension and gelatin sponge (GS) particles, has been mainly promoted in Japan, and is currently known as the conventional TACE (CTACE) and is distinguished from TACE procedures that use drug-eluting beads, the latter that is mainly promoted in Europe and the United States.

Embolization of the hepatic arteries causes tumor necrosis because these arteries feed nutrients to the HCCs. Furthermore, high concentrations of concomitantly used chemotherapeutic agents are retained in the tumors for prolonged periods of time, thus, enhancing subsequent necrosis. However, the liver parenchyma surrounding HCCs is supplied by both the arterial and portal venous circulations; therefore, it does not undergo necrosis due to arterial embolization alone. When the arterial blood flow is occluded, portal blood regurgitates into the tumor through the portal vein and surrounding sinusoids, which are the drainage veins of the tumor, to consequently facilitate tumor survival [12]. Accordingly, the incidence of local tumor recurrence after TACE is relatively high. In segmental cTACE, the reported cumulative local recurrence rates are $46 \%$ at two years, 58\% at three years, and $63 \%$ at five years, which are higher than those found in the rates reported after surgical resection or radiofrequency ablation (RFA) [13]. Better local cancer control and improved treatment outcomes have been accomplished through the use of improved catheters and advanced equipment, such as a unified computed tomography (CT) angiography system, as well as ultra-selective TACE performed for small HCCs [12].

In this article, we describe the historical course of TACE, its practical applications/methods, and the current performance status, such as treatment outcomes based on our experience, followed by a future perspective that includes attempts to improve local curability.

\section{Indications of TACE}

TACE is essential for cases of inoperable advanced HCC that are not subjected to percutaneous local therapies, particularly for hypervascular HCCs that exhibit dense staining on contrast images of the hepatic arteries. This procedure is currently performed as a standard treatment method, particularly in Japan $[6,14]$. In the early 2000s, two randomized controlled trials reported that TACE positively influenced the aforementioned antitumor effects and survival rates when compared with symptomatic treatment; however, both reports excluded Okuda class III and Child-Pugh class C patients. Furthermore, in a meta-analysis of 18 reports conducted by Camma et al., the overall two-year mortality rate was significantly lower in the TACE group than in the untreated group (odds ratio, 0.54; 95\% CI: 0.33, 0.89; $\mathrm{p}=0.015$ ) [15]. The exclusion criteria of the RCT stated that TACE for advanced HCC contributed to prognostic improvements and recommended the avoidance of cases with poor liver function, such as Okuda Stage III and Child-Pugh class C, in addition to non-selective TACE. The initial staging [14] conducted by the Barcelona Clinic Liver Cancer (BCLC) group categorizes cases as (1) Stage B (intermediate stage), $\geq 4$, Child-Pugh A-B, Okuda I-II, and performance status of $0-2$, or (2) Stage $C$ (advanced stage), with a performance status of $1-2$, the absence of portal vein invasion, lymphadenopathy or metastases. This is very similar to the 


\section{Liver}

2005 Japanese guidelines $[14,16,17]$ that described a hepatic disorder level of A or B (corresponding to the Child-Pugh classification) as 2-3 HCCs exceeding $3 \mathrm{~cm}$ and at least $4 \mathrm{~cm}$ (regardless of size) [17]. Following publication of the results of the large-scale SHARP study in 2008, which examined unresectable and advanced HCC [18], sorafenib was recommended as a standard treatment for advanced HCC. Therefore, Stage B (intermediate stage), or $\geq 4$ tumors with a Child-Pugh grade A-B and performance status of $0-2$ was determined to be the only indication of TACE/TAE in the guidelines published by the Society of Interventional Radiology (SIR) in the U.S.A. and the revised American Association for the Study of Liver Disease guidelines published by the BCLC group [19].

In June 2014, the Clinical Practice Guidelines for the Management of Hepatocellular Carcinoma, proposed by the Japan Society of Hepatology (JSH), were updated in a consensus meeting of the Liver Cancer Study Group of Japan (LCSGJ), after which the revised JSH-LCSGJ Consensus-based Treatment Algorithm for HCC was published; this revised document included a new consensus regarding suitable management of HCC in Japan [20]. This algorithm suggests that TACE is the first choice of treatment in Child-Pugh A/B patients with four or more HCCs. Even for single to less than four HCC nodules exceeding $3 \mathrm{~cm}$ in diameter, combination therapy of TACE and ablation is frequently performed when resection is not indicated. Furthermore, resection and TACE is frequently performed when portal invasion is minor such as with intraportal tumor thrombi (Vp1, Vp2). Local ablation therapy or subsegmental TACE is performed even for Child-Pugh C patients (CP score 10 and 11) within the Milan criteria, when transplantation is not indicated. However, no unified, clear eligibility and non-eligibility criteria for TACE are currently available in Japan. At our institution, the Child-Pugh classification is considered important. A liver function score of Child-Pugh A or B is considered as an indication of TACE. However, in cases with bilirubin values $>2 \mathrm{mg} / \mathrm{dL}$ or ascites even with a liver function score of Child-Pugh B, we attempt to limit the embolization area as small as possible. In principle, we do not perform either TACE or local treatment for cases with liver function scores of Child-Pugh C. We perform TACE alone for cases with multiple ( $\geq 6$ ) tumors (regardless of size) and relatively maintained liver function score of Child-Pugh A or B. In Japan, some institutions define intraportal tumor thrombi including Vp1 and Vp2 on the peripheral side of the secondary branching as indication for TACE. In addition, indications may also include cases in which percutaneous local therapy is difficult despite a small tumor size and cases in which the hepatic lesion is the prognosticator despite distant metastasis (liver dominant disease).

\section{Treatment Procedures}

The general cTACE procedures used at our hospital are described as follows.

1 Before the procedure, we conduct diagnostic imaging using ultrasonography (US), US contrast imaging, dynamic contrast-enhanced CT, and gadolinium ethoxybenzyl diethylenetriamine pentaacetic acid (Gd-EOB-DTPA)-enhanced magnetic resonance imaging (MRI) and, as required, CT angiography (CTAP: CT during arterio-portography, CTHA: CT hepatic arteriography). By combining these imaging findings, we can determine the localization and number of tumors, characteristics of the tumor(s), level of arterial flow, presence or absence and degree of invasion of the portal vein and/or bile duct, and the embolization range and level (e.g., subsegmental or segmental), as well as the drug to be injected.

2 Determination of Lipiodol dose: Determination of the type and dose of the chemotherapeutic agent, as well as the injected Lip dose, is very important because this correlates closely with the therapeutic effect and complications $[3-8,10,11,13,21]$. First, we confirm the size 
and number of tumors and quantity of tumor vessels from the preprocedure diagnostic images and intraprocedural digital subtraction angiography (DSA) and eventually determine the Lip injection dose by comprehensively considering the level reached by the catheter (e.g. we choose 5-6 $\mathrm{ml}$ of the injected dose of Lip, if the tumor is $5 \mathrm{~cm}$ in diameter, or we choose approximately $5 \mathrm{ml}$ in a segmental artery), degree of liver shrinkage and hypertrophy, and liver function, among other factors.

3 Chemotherapeutic agents: The effectiveness of all chemotherapeutic agents used in TACE for HCC have been determined [3-15, 21]. Generally, the anthracyclines including doxorubicin and epirubicin are used worldwide and in Japan, respectively. Powdered water-soluble cisplatin and fat-soluble miriplatin are also clinically available. For epirubicin, 30-60 mg are dissolved in a volume of water-soluble contrast medium equivalent to half of the Lip injection volume, followed by emulsification with Lip via mixing with 20 pumps. For cisplatin, $65 \mathrm{mg} / \mathrm{m}^{2}$ are directly mixed with Lip via ultrasonic agitation or another technique. Miriplatin, a fat-soluble platinum preparation, is also used via direct dissolution in the Lip injection dose. In some cases, a sufficient volume of miriplatin-Lip suspension cannot be injected into the tumor because the highly viscous suspension is retained inside the feeding vessel at an early point during intra-arterial injection. In such cases, the embolic material also cannot be sufficiently injected, thus potentially reducing the therapeutic effect of TACE with miriplatin. The viscosity of miriplatin can be reduced by warming it, as the ratio of viscosity at $40{ }^{\circ} \mathrm{C}$ to that at room temperature $\left(25^{\circ} \mathrm{C}\right)$ is 0.51 . Accordingly, pretreatment warming has been reported to improve the therapeutic effect of TACE $[22,23]$.

4 GS particles: The size and amount of injected GS particles are also related to the embolic effect and adverse reactions. Two different sized GS particles, with diameters of 1 and $2 \mathrm{~mm}$, are commercially available and are widely used in Japan. GS particles are injected until blood flow in the target artery is fully restricted.

5 Super-selective microcatheter insertion: In segmental TACE, accurate identification of the tumor-feeding artery and accurate catheter insertion are the keys to success. It is important to embolize with an adequate safety margin following confirmation of the relationship between dense tumor staining and the embolization area via DSA and, as required, selective CTHA after inserting a microcatheter into the arterial branch intended for embolization. We usually decide the adequate safety margin is 5 - $\mathrm{mm}$ wide for tumors $<25 \mathrm{~mm}$, and $10-\mathrm{mm}$ wide for tumors $\geq 25 \mathrm{~mm}$ [24]. We use CT or CBCT to monitor the embolization area during the procedure (Figure 1). In addition, useful software has recently been developed for a CBCT-based automated tumor-feeding vessel tracking system. Given the fact that tumorfeeding vessels can be identified with an $88 \%$ accuracy in liver malignancies of $<3 \mathrm{~cm}$ in size $[25,26]$, this technique is useful when the feeding vessel is difficult to identify.

\section{Therapeutic Efficacy of TACE}

Table 1 shows the outcomes of TACE, primarily with respect to survival rates. According to Takayasu et al., two large-scale, prospective cohort studies pertaining to factors contributing to an improved prognosis with Lip-TACE (use of Lip emulsion and GS particles) for unresectable HCCs were conducted on a nationwide scale by the Liver Cancer Study Group of Japan $[8,28]$. The maximum tumor diameter was found to be a prognostic factor. In another RCT, Lo et al. reported that a tumor diameter of $\leq 5 \mathrm{~cm}$ was a good prognostic factor [11]. The 5-year survival rate reported by Takayasu et al. was lower than that reported by Matsui et al. in 1993, even among cases involving tumors of $\leq 3 \mathrm{~cm}$ [6]. In other words, the subsegmental TACE performed by Matsui et al. had a stronger antitumor effect relative to the com- 


\section{Liver Cancer}
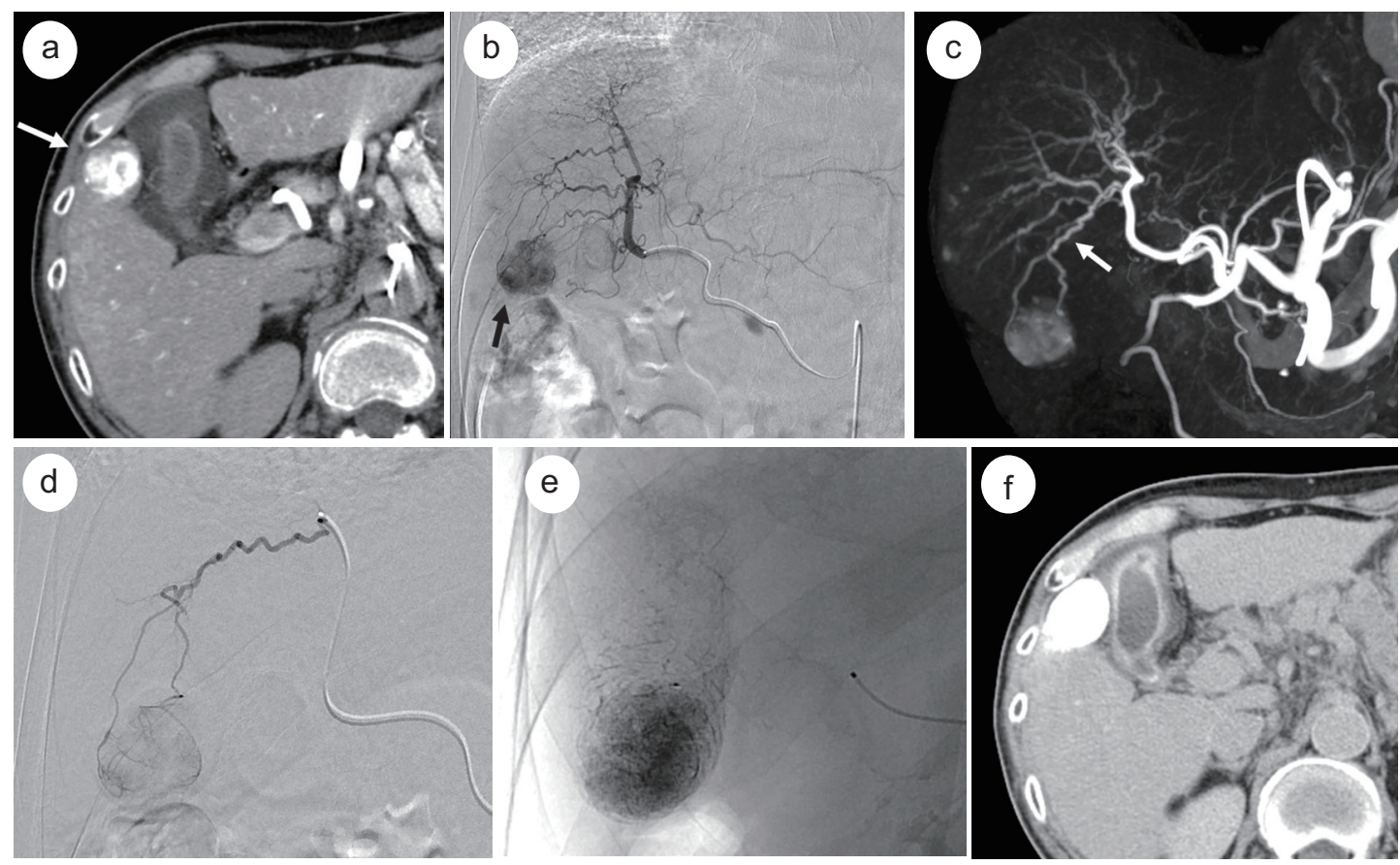

Fig. 1. 60-year-old woman with HCC. a CT hepatic arteriogram shows an enhancing nodule (arrow) in liver segment 5 . Serum alpha-fetoprotein ( $\alpha$ FP) level was $486 \mathrm{ng} / \mathrm{ml}$ (normal range; 0-5 ng/ml). b Hepatic digital subtraction arteriogram shows a hypervascular tumor nodule (arrow) in corresponding segments, 2-dimensional angiography shows an HCC as an enhanced lesion (arrow) complicated with right anterior/posterior hepatic arteries. c Maximal projection rendering image indicates a tumor-feeding artery from a right anterior hepatic artery to the tumor (arrow) according to the navigation imaging system by cone-beam CT. d Subsegmental catheterization into the feeding artery can be performed. Subsegmental TACE was performed with mixture of $2 \mathrm{ml}$ of Lipiodol and $20 \mathrm{mg}$ of epirubicin followed by gelfoam embolization. e Post-TACE plain radiograph shows better deposition of Lipiodol in the portal vein around the tumor in segment 5. f Follow-up CT scan obtained one-month later shows well-accumulated compact lipiodol retention in tumor of segment 5 without evidence of viable tumor. Serum $\alpha$ FP level was $32 \mathrm{ng} / \mathrm{ml}$.

bined so-called TACE (details of the embolization sites were unknown). Although the tumor background and differences in liver function must be considered, the technical aspect was likely reflected in this therapeutic outcome. In the latest reports, according to a large-scale tabulation of Japanese cases by Takayasu et al. [28] the survival rates of 4,966 Child-Pugh A and $\mathrm{B}$ cases were $87 \%$ at one year, $70 \%$ at two years, $55 \%$ at three years, $42 \%$ at four years, and $34 \%$ at five years, respectively. After limiting cases to Child-Pugh A and single tumors $(n=1,475)$, the rates increased to $93 \%$ at one year, $83 \%$ at two years, $73 \%$ at three years, $62 \%$ at four years, and $52 \%$ at five years, respectively (table 2). Yamakado et al. compared the prognoses between patients who underwent embolization on the peripheral side of the segmental artery $(n=703)$ and those who underwent more proximal embolization $(n=112)$ and showed that the cumulative survival rates of the former group were $83 \%$ at one year, $65 \%$ at three years, and $41 \%$ at five years, in contrast to $87 \%, 51 \%$, and $27 \%$, respectively, in the latter group, indicating that the TACE technique influences prognosis $(\mathrm{p}=0.0034)$ [27]. Takaki et al. reported that the survival rates of the subsegmental TACE group (n=93) in 1, 3, 5, 7, and 10 years were $90.3 \%, 62.9 \%, 48.1 \%, 22.5 \%$, and $12.9 \%$, respectively, whereas the corresponding rates in the segmental TACE group $(\mathrm{n}=106)$ were $92.5 \%, 66.9 \%, 30.5 \%, 18.3 \%$, and $6.1 \%$, respectively; although these findings demonstrated a better trend in the subsegmental TACE group, no significant difference was observed $(p=0.4421)$ [13]. 
Table 1. Response and Survival Rates of HCC Patients in Past Trials of TACE alone, or Comparing TACE/ TAE versus Inactive Treatments

\begin{tabular}{|c|c|c|c|c|c|c|c|c|c|}
\hline \multirow[t]{2}{*}{ Author } & \multirow[t]{2}{*}{ Year } & \multirow{2}{*}{$\begin{array}{l}\text { Study } \\
\text { Design }\end{array}$} & \multirow{2}{*}{$\begin{array}{l}\text { Treatment Arms } \\
\text { (No. of Patients) }\end{array}$} & \multirow{2}{*}{$\begin{array}{l}\text { Response } \\
\text { Rate (\%) }\end{array}$} & \multicolumn{5}{|c|}{ Survival (\%) } \\
\hline & & & & & 1-year & 2-year & 3-year & 5-year & \\
\hline Yamada [2] & 1983 & Ret. & TAE (66) & & 44 & 29 & 15 & & \\
\hline Matsui [4] & 1993 & Ret. & Subseg TACE (100) & & 100 & & 73 & 53 & \\
\hline $\begin{array}{l}\text { Nishimine } \\
{[5]}\end{array}$ & 1994 & Ret. & Seg TACE (98) & & 89 & & 59 & 30 & \\
\hline \multirow[t]{2}{*}{ GETCH [7] } & 1995 & $\mathrm{RCT}$ & TACE (50) & 16 & 62 & 38 & & & NS \\
\hline & & & Control (46) & 5 & 43 & 26 & & & \\
\hline \multirow[t]{3}{*}{ Llovet [8] } & 2002 & $\mathrm{RCT}$ & TAE (37) & 43 & 75 & 50 & 29 & & \\
\hline & & & TACE $(40)$ & 35 & 82 & 63 & 29 & & $\mathrm{P}=0.009$ \\
\hline & & & Control (35) & 0 & 63 & 27 & 17 & & \\
\hline \multirow[t]{2}{*}{ Lo [9] } & 2002 & $\mathrm{RCT}$ & TACE (40) & 27 & 57 & 31 & 26 & & $\mathrm{P}=0.002$ \\
\hline & & & Control (39) & 2.6 & 32 & 11 & 3 & & \\
\hline \multirow{4}{*}{$\begin{array}{l}\text { Takayasu } \\
{[6]}\end{array}$} & 2006 & Pros. & $<2$ cm (1989) & & 93 & & 63 & 39 & \\
\hline & & & $2.1-3 \mathrm{~cm}(1981)$ & & 90 & & 52 & 28 & \\
\hline & & & $3.1-5 \mathrm{~cm}(2318)$ & & 83 & & 43 & 23 & $P<0.001$ \\
\hline & & & $>5 \mathrm{~cm}(2076)$ & & 63 & & 30 & 16 & \\
\hline \multirow{2}{*}{$\begin{array}{l}\text { Yamakado } \\
\text { [27] }\end{array}$} & 2012 & Ret. & Seg TACE (703) & & 93 & & 65 & 41 & $\mathrm{P}=0.034$ \\
\hline & & & >Seg TACE (112) & & 87 & & 51 & 27 & \\
\hline \multirow[t]{2}{*}{ Takaki [13] } & 2012 & Ret. & Subseg TACE (93) & & 90 & & 63 & 48 & NS \\
\hline & & & Seg TACE (106) & & 93 & & 67 & 31 & \\
\hline
\end{tabular}

Seg=Segmental; Subseg=Subsegmental; TACE=transarterial chemoembolization; TAE=transarterial embolization; Ret.=retrospective study; Pros.=prospective study; RCT=randomized controlled trial; NR=Not reported; NS=not significant.

\section{Complications}

TAE/TACE has been associated with low mortality rates. According to the report by Takayasu et al., $44(0.5 \%)$ of 8,510 patients died of TACE-related causes. The causes of death included hepatic insufficiency in 18 patients, malignancy in eight patients, intraperitoneal bleeding in seven patients, gastrointestinal hemorrhage in five patients, and other causes in six remaining patients. The degree of hepatic dysfunction was grade B or C in $82 \%$ of the 44 patients [8]. Generally, post-embolization symptoms such as fever and abdominal pain develop during a period of several days after TACE, although the degree of severity varies. Other serious complications include hepatic abscess and ischemic complications (e.g. hepatic infarction, hepatic insufficiency, cholecystitis, and bile duct necrosis), for which care must be provided. In addition, in TACE procedures involving the hepatic collateral pathways that feed HCCs, care should be taken for non-target embolization. 


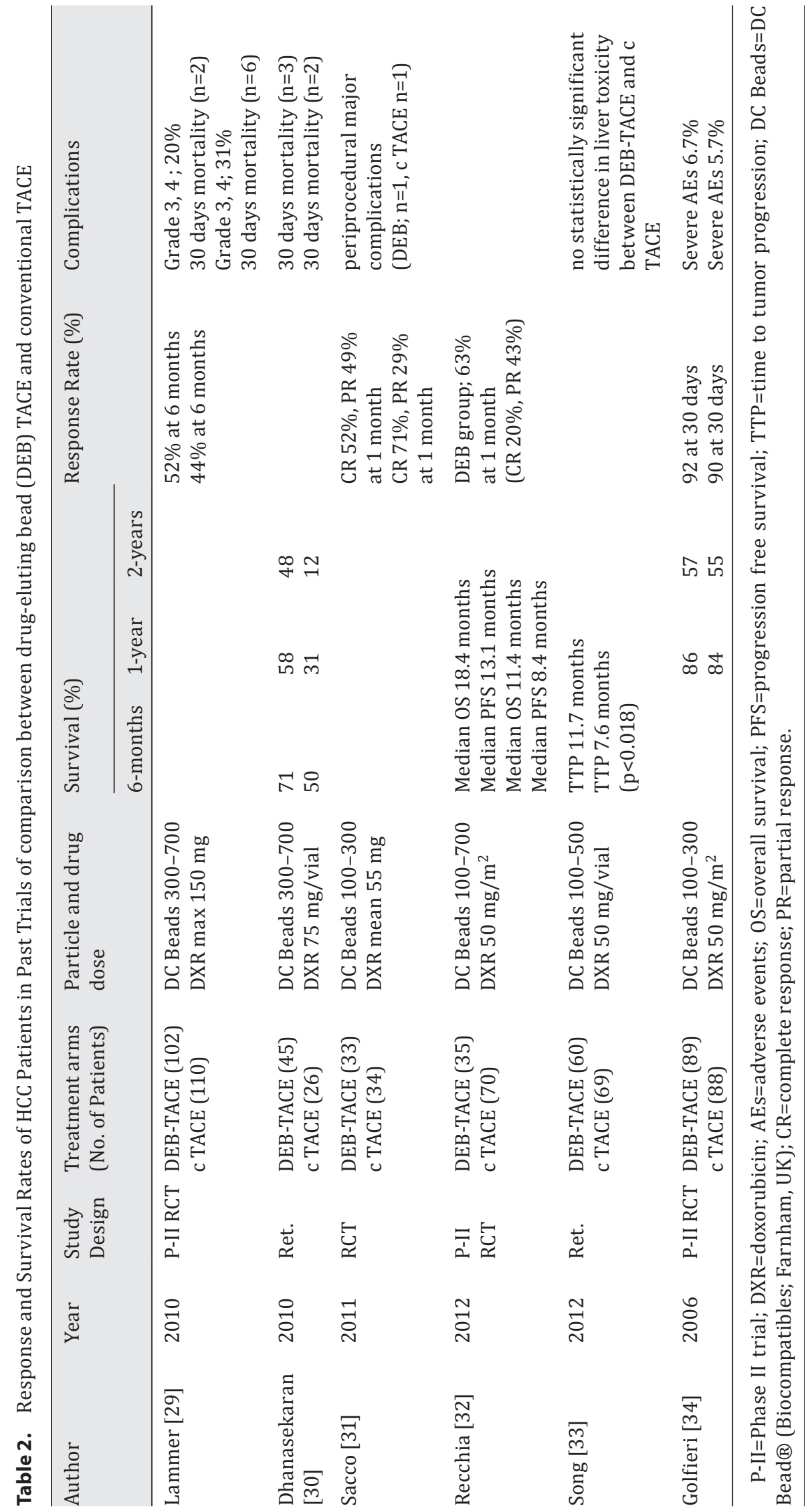




\section{Drug-Eluting Bead (DEB) TACE}

TACE procedures in Europe and the U.S.A. mainly use a non-absorptive spherical embolization substance measured in units of several hundred $\mu \mathrm{m}$ (microspheres). DEBs that adsorb and gradually release chemotherapeutic agents have been put into practice, thus conferring the role of drug carriage $[35,36]$ on the beads themselves. DEB TACE is widely used for HCC in Europe and the U.S.A.; in particular, a doxorubicin-adsorbing type of DEB called DEBDOX ${ }^{\mathrm{TM}}$ is used. Recent reports have begun to describe the 5-year survival rates associated with DEBDOX $^{\text {тм }}$. Burrel et al. reported survival rates of $89.9 \%$ at one year, $66.3 \%$ at three years, and $38.3 \%$ at five years with a median survival time of 48.6 months [37], whereas Malagari et al. reported corresponding survival rates of $93.6 \%, 62 \%, 22.5 \%$, and a median survival time of 43.8 months, respectively [38]; both studies indicate relatively good outcomes. Comparisons between DEB-TACE and Lip-TACE represent an interesting and important theme. In PRECISION-V, a Phase II RCT of DEBDOX ${ }^{\mathrm{TM}}$, the response rates at six months (primary endpoint) were $52 \%$ and $44 \%$ in the DEB-TACE and Lip-TACE groups, respectively, with no significant difference $(p=0.11)$, thus indicating the non-inferiority of DEB. However, because significant differences were detected among cases with scores of Child-Pugh B, lesions in both lobes, and disease recurrence, DEB-TACE was proposed as a useful treatment for advanced cases [29]. In an RCT published by Sacco et al., on-demand TACE was performed using an average doxorubicin dose of $55 \mathrm{mg}$, and the resulting two-year survival rates were $86.8 \%$ and $83.6 \%$ in the DEB-TACE and Lip-TACE groups, respectively; no significant difference was observed [31]. Moreover, in a recent retrospective comparison conducted by Song et al., after limiting the analysis to BCLC-B cases, the local control, time-to-tumor progression (TTP), and total survival time were superior in the DEB-TACE group [33]. All of these reports state that DEBTACE is superior with respect to sustained drug-releasing capacity and reduction of postembolization syndrome. However, there is no consensus regarding local control and survival time because of significant influences from differences in the clinical stage, hepatic sparing ability, and technical factors, among other factors.

\section{Combination Therapies with a Molecularly Targeted Drug}

TACE and sorafenib are currently standard treatments for intermediate and advancedstage HCC, respectively. Recently, there has been interest in combining antiangiogenic target agents with TACE to decrease post-TACE angiogenesis and to improve the efficacy of locoregional therapy as well as possibly decreasing the incidence of systemic disease. Therefore, some combination therapies with TACE and sorafenib have been studied after attempting combination therapies of sorafenib and adjuvant therapy/hepatic arterial infusion chemotherapy after surgical resection or RFA with expected enhancement of the therapeutic effects of surgical resection/local therapy [39].

To clarify the usefulness of sorafenib as a post-TACE adjuvant therapy, a placebo-controlled, randomized comparative study was conducted (post-TACE study). Sorafenib was administered to TACE-treated patients with HCC as a post-TACE adjuvant therapy until cancer progression was observed. However, the usefulness of sorafenib could not be demonstrated because no significant differences were observed between the sorafenib $(\mathrm{n}=229)$ and placebo groups ( $n=229)$ in the TTP [5.4 vs. 3.7 months; hazard ratio, $0.87(0.70-1.09) ; p=0.252$ ] and survival time [29.7 days vs. not reached; hazard ratio, $1.06(0.69-1.64) ; p=0.790)$ [40]. As combination therapy was expected to prolong the time until TACE became clinically unusable, a randomized Phase II study was conducted in which TACE-sorafenib combination therapy and TACE-placebo were compared (sPACE study). Although the TTPs (primary end- 
point) were 5.6 months and 5.5 months in the sorafenib and placebo groups, respectively, with a hazard ratio of $0.797(0.588-1.080 ; \mathrm{p}=0.072)$, TTP was reported to be significantly longer in the sorafenib group because the significance level had been set at $15 \%$. However, the study results do not allow us to state that the significant effect of TACE-sorafenib combination therapy was sufficiently clear because the time until TACE became clinically unusable [ $95 \mathrm{vs.}$ 224 days; hazard ratio, 1.586 (1.200-2.096); $\mathrm{p}=0.999$ ] was better in the placebo group and no significant difference was observed in the survival time [hazard ratio, $0.898(0.606-1.330)$; $\mathrm{p}=0.295][41,42]$.

Currently ongoing studies of TACE-sorafenib combination therapies include the TACE-2 study (NCT01324076) and ECOG1208 study (NCT01004978), which are being conducted in Europe and the U.S.A., as well as the TACTICS study (NCT01217034) being conducted in Japan. Therefore, clarification of the significance of concomitant sorafenib and TACE is expected in the future.

\section{Conclusion and Future Perspectives}

Although BCLC-stage B disease is internationally considered a good indication of TACE, BCLC-stage B categorizes cases as tumor numbers $\geq 4$, Child-Pugh A-B, and performance status of $0-2$. Therefore, a movement to determine whether DEB-TACE or Lip-TACE is more appropriate through clarification of the conditions in which the respective advantages are clear. Conversely, intermediate-stage (BCLC-stage B) HCC includes a heterogeneous population of patients with varying tumour burdens, liver function and disease etiology. Therefore, not all patients with intermediate-stage HCC may derive similar benefit from TACE, and some patients may benefit from other locoregional (e.g. RFA) or medical treatment with sorafenib. Some clinical trials aimed at investigating the potential role of this molecule in the treatment of patients with intermediate-stage HCC within combination therapeutic regimens are ongoing.

The efficacy of DEB-TACE for Lip-TACE-resistant advanced HCC is also promising. Sorafenib therapy has been conventionally performed as a standard treatment for Lip-TACEresistant advanced HCC, according to a subanalysis of RCTs (SHARP trial and Asia-Pacific trial) conducted mainly in Europe, the U.S.A. and Asia [43, 44]. At present, DEB-TACE is not positioned as a replacement for Lip-TACE because no significant results were obtained in RCTs of DEB-TACE in Europe and the U.S.A. However, DEB-TACE may become a new treatment option for advanced HCC that has become refractory to Lip-TACE [45-47]. DEB-TACE is likely to have distinct pharmacokinetics/action mechanisms because in comparison to Lip, DEB can maintain higher levels of chemotherapeutic agent within tumors because of slower sustained agent release, and can thus reduce the peripheral blood level. Accordingly, DEB-TACE might be effective against Lip-TACE-resistant advanced HCC [47]. Currently, a multicenter Phase II study to determine the safety and efficacy of DEB-TACE for Lip-TACE-resistant advanced HCC is ongoing in Japan (Phase II trial of Transarterial Chemoembolization using Drug-eluting bead for advanced HCC refractory to Lipiodol-TACE: TALENT study, now registered NCI), and therefore we await further developments to determine whether DEB-TACE can potentially replace Lip-TACE as a standard therapy and to investigate the different applications of DEBs.

\section{Conflict of Interest}

The authors declare no conflict of interest. 


\section{References}

1 Jemal A, Bray F, Center MM, Ferlay J, Ward E, Forman D: Global cancer statistics. CA Cancer J Clin 2011;61:69-90.

2 Yamada R, Sato M, Kawabata M, Nakatsuka H, Nakamura K, Takashima S: Hepatic artery embolization in 120 patients with unresectable hepatoma. Radiology 1983;148:397-401.

3 Nakamura H, Hashimoto T, Oi H, Sawada S: Transcatheter oily chemoembolization of hepatocellular carcinoma. Radiology 1989;170:783-786.

4 Uchida H, Ohishi H, Matsuo N, Nishimine K, Ohue S, Nishimura Y, Maeda M, Yoshioka T: Transcatheter hepatic segmental arterial embolization using lipiodol mixed with an chemotherapeutic drug and Gelfoam particles for hepatocellular carcinoma. Cardiovasc Intervent Radiol 1990;13:140-145.

5 Nakao N, Kamino K, Miura K, Takayasu Y, Ohnishi M, Miura T: Transcatheter arterial embolization in hepatocellular carcinoma: a long-term follow-up. Radiat Med 1992;10:13-18.

6 Matsui O, Kadoya M, Yoshikawa J, Gabata T, Arai K, Demachi H, Miyayama S, Takashima T, Unoura M, Kogayashi K: Small hepatocellular carcinoma: treatment with subsegmental transcatheter arterial embolization. Radiology 1993;188:79-83.

7 Nishimine K, Uchida H, Matsuo N, Sakaguchi H, Hirohashi S, Nishimura Y, Guo Q, Ohishi H, Nagano N, Yoshioka T, et al: Segmental transarterial chemoembolization with Lipiodol mixed with chemotherapeutic drugs for nonresectable hepatocellular carcinoma: follow-up CT and therapeutic results. Cancer Chemother Pharmacol 1994;33(Suppl):S60-S68.

8 Takayasu K, Arii S, Ikai I, Omata M, Okita K, Ichida T, Matsuyama Y, Nakanuma Y, Kojiro M, Makuuchi M, Yamaoka Y, Liver Cancer Study Group of Japan: Prospective cohort study of transarterial chemoembolization for unresectable hepatocellular carcinoma in 8510 patients. Gastroenterology 2006;131:461-469.

9 Groupe d'Etude et de Traitement du Carcinome Hépatocellulaire: A comparison of lipiodol chemoembolization and conservative treatment for unresectable hepatocellular carcinoma. N Engl J Med 1995;332:1256-1261.

10 Llovet JM, Real MI, Montaña X, Planas R, Coll S, Aponte J, Ayuso C, Sala M, Muchart J, Solà R, Rodés J, Bruix J, Barcelona Liver Cancer Group: Arterial embolisation or chemoembolisation versus symptomatic treatment in patients with unresectable hepatocellular carcinoma: a randomised controlled trial. Lancet 2002;359:1734-1739.

11 Lo CM, Ngan H, Tso WK, Liu CL, Lam CM, Poon RT, Fan ST, Wong J: Randomized controlled trial of transarterial lipiodol chemoembolization for unresectable hepatocellular carcinoma. Hepatology 2002;35:11641171.

12 Ekelund L, Lin G, Jeppsson B: Blood supply of ex-perimental liver tumors after intraarterial embolization with Gelfoam powder and absolute ethanol. Cardiovasc Intervent Radiol 7: 234-239, 1984.

13 Takaki S, Sakaguchi H, Anai H, et al.: Long-term outcome of transcatheter subsegmental and segmental arterial chemoembolization using lipiodol for hepatocellular carcinoma. Cardiovasc lntervent Radiol 35: 544-554, 2012.

14 Bruix J, Sala M, Llovet JM: Chemoembolization for hepatocellular carcinoma. Gastroenterology 2004;127(Suppl 1):S179-S188.

15 Cammà C, Schepis F, Orlando A, Albanese M, Shahied L, Trevisani F, Andreone P, Craxì A, Cottone M: Transarterial chemoembolization for unresectable hepatocellular carcinoma: meta-analysis of randomized controlled trials. Radiology 2002;224:47-54.

16 Llovet JM: Updated treatment approach to hepatocellular carcinoma. J Gastroenterol 2005;40:225-235.

17 Makuuchi M, Kokudo N, Arii S, Futagawa S, Kaneko S, Kawasaki S, Matsuyama Y, Okazaki M, Okita K, Omata M, Saida Y, Takayama T, Yamaoka Y: Development of evidence-based clinical guidelines for the diagnosis and treatment of hepatocellular carcinoma in Japan. Hepatol Res 2008;38:37-51.

18 Llovet JM, Ricci S, Mazzaferro V, Hilgard P, Gane E, Blanc JF, de Oliveira AC, Santoro A, Raoul JL, Forner A, Schwartz M, Porta C, Zeuzem S, Bolondi L, Greten TF, Galle PR, Seitz JF, Borbath I, Häussinger D, Giannaris T, Shan M, Moscovici M, Voliotis D, Bruix J, SHARP Investigators Study Group: Sorafenib in advanced hepatocellular carcinoma. N Engl J Med 2008;359:378-390.

19 Bruix J, Sherman M, American Association for the Study of Liver Diseases: Management of hepatocellular carcinoma: an update. Hepatology 2011;53:1020-1022.

20 Kudo M, Matsui O, Izumi N, et al: JSH Consensus-Based Clinical Practice Guidelines for the Management of Hepatocellular Carcinoma: 2014 Update by the Liver Cancer Study Group of Japan. Liver Cancer 2014;3:458-468.

21 Miyayama S, Matsui O, Yamashiro M. et al.:Ultraselective transcatheter arterial chemoembolization with a2-f tip microcatheter for small hepatocellular carcinomas: Relationship between local tumor recurrence and visualization of the portal vein with iodized oil. J Vasc Interv Radiol 18: 365-376. 2007.

22 Kora S, Urakawa H, Mitsufuji T, el al.:Warming effect of miliplatin-lipodol suspension as a chemotherapeutic agent for transarterial chemoembolization for hepatocellular carcinoma: preliminary clinical experience. Cardiovasc Inlervent Radiol 36: 1023-1029.2013.

23 Seko Y, Ikeda K, Kawamura Y, Fukushima T, Hara T, Sezaki H, Hosaka T, Akuta N, Suzuki F, Kobayashi M, Suzuki Y, Saitoh S, Arase Y, Kumada H: Antitumor efficacy of transcatheter arterial chemoembolization with warmed miriplatin in hepatocellular carcinoma. Hepatol Res 2013;43:942-949. 
24 Miyayama S, Yamashiro M, Hashimoto M, Hashimoto N, Ikuno M, Okumura K, Yoshida M, Matsui O: Comparison of local control in transcatheter arterial chemoembolization of hepatocellular carcinoma $\leq 6 \mathrm{~cm}$ with or without intraprocedural monitoring of the embolized area using cone-beam computed tomography. Cardiovasc Intervent Radiol 2014;37:388-395.

25 Minami Y, Yagyu Y, Murakami T, Kudo M: Tracking Navigation Imaging of Transcatheter Arterial Chemoembolization for Hepatocellular Carcinoma Using Three-Dimensional Cone-Beam CT Angiography. Liver Cancer 2014;3:53-61.

26 Miyayama S, Yamashiro M, Hashimoto M, Hashimoto N, Ikuno M, Okumura K, Yoshida M, Matsui O: Identification of small hepatocellular carcinoma and tumor-feeding branches with cone-beam CT guidance technology during transcatheter arterial chemoembolization. J Vasc Interv Radiol 2013;24:501-508.

27 Yamakado K, Miyayama S, Hirota S. et al.:Hepatic arterial embolization for unresectable hepatocellular carcinomas: do technical factors affect prognosis? Jpn J Radio1 30: 560-566,2012.

28 Takayasu K, Arij S, Kudo M, et al.:Superselective transarterial chemoembolization for hepatocellular carcinoma.validation of treatment algorithm proposed by Japanese guidelines. J Hepato1 56;886-892,2012.

29 Lammer J, Malagari K, Vogl T, et al. Prospective randomized study of doxorubicin-eluting-bead embolization in the treatment of hepatocellular carcinoma: results of the PRECISION V study. Cardiovasc Intervent Radio1 33: 41-52, 2010.

30 Dhanasekaran R, Kooby DA, Staley CA, Kauh JS, Khanna V, Kim HS: Comparison of conventional transarterial chemoembolization (TACE) and chemoembolization with doxorubicin drug eluting beads (DEB) for unresectable hepatocelluar carcinoma (HCC). J Surg Oncol 2010;101:476-480.

31 Sacco R, Bargellini I, Bertini M, et al. Conventional versus doxorubicin-eluting bead transarterial chemoembolization for hepatocellular carcinoma. J Vasc lnterv Radio1 22: 1545-1552, 2011.

32 Recchia F, Passalacqua G, Filauri P, Doddi M, Boscarato P, Candeloro G, Necozione S, Desideri G, Rea S: Chemoembolization of unresectable hepatocellular carcinoma: Decreased toxicity with slow-release doxorubicin-eluting beads compared with lipiodol. Oncol Rep 2012;27:1377-1383.

33 Song MJ, Chun HJ, Song S, Kim HY, Yoo SH, Park CH, Bae SH, Choi JY, Chang UI, Yang JM, Lee HG, Yoon SK: Comparative study between doxorubicin-eluting beads and conventional transarterial chemoembolization for treatment of hepatocellular carcinoma. J Hepatol 2012;57:1244-1250.

34 Golfieri R, Giampalma E, Renzulli M, Cioni R, Bargellini I, Bartolozzi C, Breatta AD, Gandini G, Nani R, Gasparini D, Cucchetti A, Bolondi L, Trevisani F, PRECISION ITALIA STUDY GROUP: Randomised controlled trial of doxorubicin-eluting beads vs conventional chemoembolisation for hepatocellular carcinoma. Br J Cancer 2014;111:255-264.

35 Liapi E, Lee KH, Georgiades CC, Hong K, Geschwind JF: Drug-eluting particles for interventional pharmacology. Tech Vasc Interv Radiol 2007;10:261-269.

36 Malagari K: Drug-eluting particles in the treatment of HCC: chemoembolization with doxorubicin-loaded DC Bead. Expert Rev Chemotherapeutic Ther 2008;8:1643-1650.

37 Burrel M, Reig M, Forner A, Barrufet M, de Lope CR, Tremosini S, Ayuso C, Llovet JM, Real MI, Bruix J: Survival of patients with hepatocellular carcinoma treated by transarterial chemoembolisation (TACE) using Drug Eluting Beads. Implications for clinical practice and trial design. J Hepatol 2012;56:1330-1335.

38 Malagari K, Pomoni M, Moschouris H, et al. Chemoembolization with doxorubicin-eluting beads for unresectable hepatocellular carcinoma: five-year survival analysis. Cardiovasc Intervent Radio1 35: 1119 $1128,2012$.

39 Kim HY, Park JW: Clinical trials of combined molecular targeted therapy and locoregional therapy in hepatocellular carcinoma: past, present, and future. Liver Cancer 2014;3:9-17.

40 Kudo M, Imanaka K, Chida N, Nakachi K, Tak WY, Takayama T, Yoon JH, Hori T, Kumada H, Hayashi N, Kaneko S, Tsubouchi H, Suh DJ, Furuse J, Okusaka T, Tanaka K, Matsui O, Wada M, Yamaguchi I, Ohya T, Meinhardt G, Okita K: Phase III study of sorafenib after transarterial chemoembolisation in Japanese and Korean patients with unresectable hepatocellular carcinoma. Eur J Cancer 2011;47:2117-2127.

41 Lencioni R, Llovet JM, Han G, et al. Sorafenib or placebo in combination with transarterial chemoembolization (TACE) with doxorubicin-eluting beads (DEBDOX) for intermediate-stage hepatocellular carcinoma (HCC): Phase II, randomized, double-blind SPACE trial. J Clin Oncol. 2012;30 (suppl 4; abstr LBA154).

42 Lencioni R: Chemoembolization in patients with hepatocellular carcinoma. Liver Cancer 2012;1:41-50.

43 Bruix J, Raoul JL, Sherman M, Mazzaferro V, Bolondi L, Craxi A, Galle PR, Santoro A, Beaugrand M, Sangiovanni A, Porta C, Gerken G, Marrero JA, Nadel A, Shan M, Moscovici M, Voliotis D, Llovet JM: Efficacy and safety of sorafenib in patients with advanced hepatocellular carcinoma: subanalyses of a phase III trial. J Hepatol 2012;57:821-829.

44 Cheng AL, Guan Z, Chen Z, Tsao CJ, Qin S, Kim JS, Yang TS, Tak WY, Pan H, Yu S, Xu J, Fang F, Zou J, Lentini G, Voliotis D, Kang YK: Efficacy and safety of sorafenib in patients with advanced hepatocellular carcinoma according to baseline status: subset analyses of the phase III Sorafenib Asia-Pacific trial. Eur J Cancer 2012;48:1452-1465.

45 Lewis AL, Taylor RR, Hall B, Gonzalez MV, Willis SL, Stratford PW: Pharmacokinetic and safety study of doxorubicin-eluting beads in a porcine model of hepatic arterial embolization. J Vasc Interv Radiol 2006;17:1335-1343.

46 Vogl TJ, Lammer J, Lencioni R, Malagari K, Watkinson A, Pilleul F, Denys A, Lee C: Liver, gastrointestinal, and cardiac toxicity in intermediate hepatocellular carcinoma treated with PRECISION TACE with drugeluting beads: results from the PRECISION V randomized trial. AJR Am J Roentgenol 2011;197:W562-570.

47 Raoul JL, Gilabert M, Piana G: How to define transarterial chemoembolization failure or refractoriness: a European perspective. Liver Cancer 2014;3:119-124. 